ERRATUM

\title{
Multiple endocrine neoplasia type 2 syndromes (MEN 2): results from the ItaMEN network analysis on the prevalence of different genotypes and phenotypes
}

Cristina Romei, Stefano Mariotti ${ }^{1}$, Laura Fugazzola ${ }^{2}$, Augusto Taccaliti ${ }^{3}$, Furio Pacini ${ }^{4}$, Giuseppe Opocher ${ }^{5}$, Caterina Mian ${ }^{6}$, Maurizio Castellano ${ }^{7}$, Ettore degli Uberti ${ }^{8}$, Isabella Ceccherini ${ }^{9}$, Nadia Cremonini ${ }^{10}$, Ettore Seregni $^{11}$, Fabio Orlandi ${ }^{12}$, Piero Ferolla ${ }^{13}$, Efisio Puxeddu ${ }^{13}$, Francesco Giorgino ${ }^{14}$, Annamaria Colao ${ }^{15}$, Paola Loli ${ }^{16}$, Fabio Bondi ${ }^{17}$, Barbara Cosci, Valeria Bottici, Antonello Cappai ${ }^{1}$, Giovanni Pinna ${ }^{1}$, Luca Persani ${ }^{2}$, Verga Uberta $^{2}$, Marco Boscaro ${ }^{3}$, Maria Grazia Castagna ${ }^{4}$, Carlo Cappelli ${ }^{7}$, Maria Chiara Zatelli ${ }^{8}$, Antongiulio Faggiano $^{15}$, Giuseppe Francia ${ }^{18}$, Maria Luisa Brandi ${ }^{19}$, Alberto Falchetti ${ }^{19}$, Aldo Pinchera, Rossella Elisei and The ItaMEN network

The journal and the authors apologise for an error in the name of one of the authors (appears as Verga Uberta) of this article published in the European Journal of Endocrinology Vol 163 301-308. The correct name of the author should be Uberta Verga and not as published.

European Journal of Endocrinology 163963 